\title{
Canetti, Yovkov et la plaine danubienne, ou le " métier du poète » face aux frontières
}

Lioubov Savova

\section{(2) OpenEdition}

1 Journals

Édition électronique

URL : https://journals.openedition.org/cher/7974

DOI : $10.4000 /$ cher.7974

ISSN : 2803-5992

Éditeur

Presses universitaires de Strasbourg

\section{Édition imprimée}

Date de publication : 1 décembre 2009

Pagination : 53-68

ISBN : 978-2-35410-006-3

ISSN : 1968-035X

\section{Référence électronique}

Lioubov Savova, «Canetti, Yovkov et la plaine danubienne, ou le « métier du poète » face aux frontières », reCHERches [En ligne], 3 | 2009, mis en ligne le 15 décembre 2021, consulté le 17 décembre 2021. URL : http://journals.openedition.org/cher/7974; DOI : https://doi.org/10.4000/cher 7974

\section{cc) (ㅇㅇㅇ}

Ce(tte) œuvre est mise à disposition selon les termes de la Licence Creative Commons Attribution Pas d'Utilisation Commerciale - Partage dans les Mêmes Conditions 4.0 International. 


\title{
Canetti, Yovkov et la plaine danubienne, ou le "métier du poète » face aux frontières
}

\author{
LIOUBOV SAVOVA \\ Paris 3 - Sorbonne Nouvelle
}

a figure du passant - un homme proche des figurines de Giacometti,
saisies dans l'instant prolongé de leur marche inlassable - est une image en mouvement qui convient fort bien à Elias Canetti (1905-1994) et Yordan Yovkov (1880-1937). Dans le sillage de Giacometti, et avec la même exigence d'un dépaysement de la pensée et des sensibilités, Canetti aura imaginé à son tour la figure d'un homme étrange - celui qui «court le long des frontières et maudit leur étanchéité» (Canetti, 1985 : 1331).

Canetti s'est toujours revendiqué un Dichter - poète et écrivain en allemand. La même force vive de l'étymologie sera au cœur de notre lecture: tel le poète, l'écrivain est un créateur d'espaces. Aussi, l'itinéraire croisé de ces deux grands auteurs du $\mathrm{xx}^{\mathrm{e}}$ siècle européen (et le regard qu'ils posent sur la plaine du Danube), nous invite à faire une expérience de la frontière dense et nuancée, dès l'enfance, qui se traduit par une résistance à la logique militaire pendant les années de guerre, avant de devenir la matrice et le prototype d'un "métier du poète», capable de transformer la ligne-frontière en espace littéraire.

Pour Canetti, né à Roustchouk sur les berges du Danube, ce fleuve est moins une frontière qu'une invitation au voyage - voyage géographique, culturel et linguistique, mais toujours projeté sur la sphère de l'intime. Bien complexe est la «carte d'identité » de Canetti, en effet: citoyen britannique de langue allemande, à passeport turc, né en Bulgarie dans une famille de juifs sépharades. Il y a d'abord chez lui l'enchantement de la diversité humaine: 
Roustchouk, sur le Danube inférieur, où je suis venu au monde, était une ville merveilleuse pour un enfant, et si je me bornais à la situer en Bulgarie, on s'en ferait à coup sûr une idée tout à fait incomplète: des gens d'origines diverses vivaient là et l'on pouvait entendre parler sept ou huit langues différentes dans la journée. Hormis les Bulgares [...], il y avait beaucoup de Turcs qui vivaient dans un quartier bien à eux, et, juste à côté, le quartier des sépharades espagnols, le nôtre. (Canetti, 1985)

Sans cet émerveillement de l'étrangeté sous toutes ses formes, la vie de Canetti-écrivain resterait incomprise. Dans un célèbre chapitre intitulé «Orgueil familial», n'avait-il pas tant insisté sur la valeur qu'avait eue cette ville du Danube à ses yeux d'enfant, n'hésitant pas à y trouver la quintessence de son expérience européenne par la suite?

Comme ville portuaire sur le Danube, Roustchouk avait eu une certaine importance dans le passé. Le port avait attiré des gens de partout et il était constamment question du Danube. [...]

Je n'arriverais sans doute pas à évoquer d'une manière satisfaisante les riches couleurs de ces premières années à Roustchouk, [...]. Rien de ce que je vivrai plus tard qui ne se fût déjà produit, sous une forme ou sous une autre, à Roustchouk, en ce temps là. L'Europe, là, c'était le reste du monde. Quand quelqu'un remontait le Danube vers Vienne, on disait: il va en Europe; l'Europe commençait là où finissait autrefois l'Empire ottoman. (Canetti, 1985)

L'enfant ne sait pas vraiment ce qu'est une frontière, ou plutôt se repère-t-il selon les délimitations que lui indiquent les adultes alentour. Ce qu'un enfant perçoit, en revanche, ce sont les frontières entre les êtres, mais il est ébahi devant la diversité:

Enfant, je n'avais pas une vision d'ensemble de cette multiplicité mais j'en ressentais constamment les effets. Certains personnages sont restés gravés dans ma mémoire uniquement parce qu'ils appartenaient à des ethnies particulières, se distinguant des autres par leur tenue vestimentaire. (Caneti, 1985)

Une seule exigence restera constantechez Canetti: ne pas fermer sa sensibilité aux autres, ne pas rester aveugle, aveuglé par les enjeux idéologiques, mais savoir se fausser compagnie, en quelque sorte, afin de rester éveillé à la diversité, pouvoir accueillir même le plus «infime» étranger.

Pour brièvement le présenter, Yordan Yovkov (1880-1937) fait partie de la génération née au lendemain de la défaite de l'Empire ottoman dans ses provinces balkaniques. C'est par la finesse de son art du récit que Yovkov s'est imposé dans cette littérature jeune, dans un pays qui passe de sa période 
de Réveil national à la Modernité. L'art de conteur, chez lui, allie une fine connaissance psychologique des personnages à la plasticité de la description. Esquisse vivante, le paysage yovkovien respire tel un personnage, se brise ou répond à ses échos - grinçants, mélancoliques ou tendres.

Deux sont les pôles d'attache chez Yovkov: sa Jéravna natale, et la Dobroudja. Le premier récit qu'il publie, «La Plainte du berger» (1910) est dédié à Jéravna: avec lui, Yovkov pose la pierre angulaire de son célèbre recueil Les Légendes de la Stara Planina (qu'il reprendra durant ses années d'exil à Bucarest, 1920-1927). Quant à la Dobroudja, l'urgence de son sort en tant que territoire perdu transforme Yovkov en chroniqueur militaire: une bonne part de ses Chroniques 1912-1918 est "dobroudjane», qui fait connaître l'écrivain à un public plus large, tout en suspendant le cycle sur Jéravna.

Mais la frontière, dès le plus jeune âge de Yovkov, se confond avec une perception intime, visuelle et affective. Dans un dialogue des voix comme dans une fugue, la frontière entre le dehors et le dedans devient perméable: elle se dessine là où disparaît de sa vue une personne aimée, son père. C'est bien à Jéravna que Yovkov fera sa première expérience de la ligne séparatrice: son père Stéphane Yovkov, berger de son état, devait suivre les transhumances ${ }^{1}$, et faisait la navette entre ce village du Balkan et la plaine du Danube.

En suivant des yeux son père s'éloigner de Jéravna en direction de la plaine du Danube, le jeune Yovkov charge déjà la Dobroudja d'un désir particulier, qu'on pourrait nommer une "métonymie affective»: la figure affectionnée et le lieu forment un tout dynamique, fortement chargé2.

Ainsi, l'investissement affectif de Yovkov face à la Dobroudja perdue comporte plusieurs couches au moment de l'annexion roumaine: la Dobroudja perdue est à la fois le lieu rêvé de l'enfance, le nouveau foyer de l'adolescence, et le seul foyer de l'homme mûr.

1 Par le rythme régulier de ces apparitions/disparitions par-delà le massif, le père de Yovkov n'a-t-il pas forgé chez son fils cette perception de la ligne frontière - une déchirure qui ne dit pas son nom, mais dont l'écrivain reprendra le thème (en le transformant) dans ses récits ultérieurs?

2 En 1897, la famille de Yovkov quitte définitivement Jéravna, appelée par le père dans la nouvelle demeure qu'il a construite pour eux en Dobroudja (à Tchifoutköy, aujourd'hui Yovkovo). Et si Yovkov devra s'absenter de ce village danubien pour ses études à Sofia, c'est bien la Dobroudja qui devient son nouveau foyer. A la mort du père (1903), l'écrivain abandonne ses études pour y revenir. Il sillonnera la plaine pendant dix ans en tant qu'instituteur. 
Et de même que Vienne, avant même qu'il la connaisse, aura été pour Canetti cette ville merveilleuse que les rêveries nostalgiques de ses parents lui léguaient, de même, le temps passé à Jéravna est pétri dans l'attente pour Yovkov, nourri d'un désir de l'ailleurs, et c'est la Dobroudja qui l'incarne.

Ce n'est qu'en considérant la proportion de l'attachement préalable de l'enfant à ses lieux, que l'on peut apprécier la véritable valeur du geste d'écrivain, geste de résistance chez Yovkov et Canetti face aux frontières érigées, leur refus à soumettre leur plume à la seule revendication territoriale.

C'est sans doute la raison pour laquelle, à l'heure des frontières militairement ou idéologiquement interdites, Canetti comme Yovkov ressentent-ils ce déchirement singulier dont parle Peter Handke, lorsqu'il en vient à distinguer le regard d'un touriste sur l'espace, de celui du véritable passeur de frontières: "Si tu ressens la douleur des seuils, c'est que tu n'es pas un touriste et le passage peut avoir lieu.» (Handke, 1987).

Mais quel seuil devra-t-il être franchi, en ces années-là?

Rappelons brièvement les faits.

La Dobroudja au sens large comprend les territoires au sud et au nord de l'embouchure du Danube près de la mer Noire. Riche sur le plan agricole, cette région est un point stratégique important, ce qui la place au cœur de nombreux conflits. Ainsi la question de la Dobroudja se pose-t-elle déjà à la fin de la guerre russo-turque de 1877-1878: les terres sont alors divisées en Dobroudja du Nord (roumaine), et Dobroudja du Sud (bulgare). Plus tard, en juillet-août 1913, la Roumanie envahit la Bulgarie (occupée à guerroyer sur ses frontières serbes et grecques), et la partie sud de la Dobroudja est annexée ${ }^{3}$. Des milliers de Dobroudjans sont confrontés à un dilemme: abandonner leurs foyers pour rejoindre le territoire bulgare; ou bien rester sur place et conserver leurs terres, mais opter pour la nationalité roumaine, avec toutes les conséquences que cela suppose ${ }^{4}$.

3 Pour mémoire: on découpe alors la frontière roumano-bulgare selon un nouveau tracé. Sur le Danube, cette nouvelle frontière fait basculer en territoire roumain des villes bulgares importantes comme Dobritch, Silistra et Toutrakan, et s'arrête près de Roustchouk. Il faudra attendre 1947 pour que le sud dobroudjane redevienne bulgare, mais Yovkov n'aura pas connu ce jour.

4 D'après Antonina Kuzmanova, historienne de la Dobroudja, les écoles bulgares sont fermées. L'annexion s'accompagne de mesures administratives visant la «roumanisation » rapide du territoire (voir Kuzmanova, 1987). 
Soldat puis correspondant du front pendant la Grande Guerre, Yovkov sillonne les frontières et décrit la migration des Bulgares de la Dobroudja, que l'annexion a rendus indésirables.

Curieusement, c'est de ce même mouvement de frontière bulgaroroumaine que devra plus tard témoigner Elias Canetti, lorsqu'il publie le premier tome de son autobiographie, La Langue sauvée! Vue par un enfant de dix ans à l'époque (1915), racontée bien plus tard par l'écrivain (1977), la frontière mouvante de la Dobroudja quitte le seul univers militaire des Chroniques de Yovkov, pour être confiée à un souvenir d'autant plus émouvant, qu'impartial et distancié.

«II ne faisait pas bon traverser les frontières» (Canetti)

«Dans les Balkans, il ne faisait pas bon traverser les frontières», remarque posément Canetti dans La Langue sauvée. Mais ces mots pourraient être étendus à bon nombre de ses expériences de passeur de frontières européen, en ces années de guerre, et permettraient de mieux saisir les enjeux du passage dans les Chroniques 1912-1918 de Yovkov. Le ton de Canetti a beau être plus détaché que celui de Yovkov, les épisodes ne provoquent pas moins, chez le lecteur, un rire jaune.

L'été 1915, le jeune Elias fait un voyage en Bulgarie en compagnie de sa mère. C'est le pays où il avait vécu jusqu'en 1911, mais que la famille avait quitté pour Manchester, avant de s'installer à Vienne. C'est donc la première fois qu'Elias et sa mère y retournent depuis. Ils décident de revoir le château d'Euxinograd, mais il y a surprise:

De Varna, on partit en excursion à Monastir, non loin d'Euxinograd, où se trouve un château royal. On ne put voir le château que de loin. Depuis la fin de la deuxième guerre des Balkans - peu de temps en somme -, le château ne se trouvait plus en Bulgarie mais en Roumanie. Dans les Balkans, après les guerres très dures qui s'y étaient déroulées, il ne faisait pas bon traverser des frontières; d'ailleurs, la plupart des postes étaient fermés et ceux qui étaient ouverts, on avait plutôt tendance à les éviter. (Canetti, 1985: 122sq, nous soulignons)

Bien que cette mention par Canetti de la route barrée n'excède pas, dans la tonalité ni la longueur, la simple notation, le fait même de la rapporter est parlant: l'enfant Elias n'a pas été indifférent à cette coupure extraordinaire, pas plus que l'écrivain ne l'est, soixante ans plus tard, lorsqu'il conclut: «Dans les Balkans [...] il ne faisait pas bon traverser les frontières», est une 
phrase qui se lit à la fois comme un regret et comme une désapprobation marquée délicatement.

Lorsque le regard de Canetti enfant s'attarde sur les mêmes aberrations de l'Histoire que celui de Yovkov, écrivain bulgare de trente-cinq ans, l'éclairage n'est pas le même que si le texte de Yovkov était lu isolément ${ }^{5}$. On relève l'étonnement de Canetti devant l'épisode de cette coupure, qu'il découvre à peine: mais s'il en garde le souvenir, si, plus de soixante ans après, Canetti l'évoque encore, c'est qu'entre-temps il s'est beaucoup intéressé à ce qui fait frontière entre les hommes d'où qu'ils viennent, à commencer par ceux dans les Balkans.

C'est sur le chemin du retour vers Vienne, en ce même été 1915, que Canetti fait l'expérience de ce qu'il appellera «la haine entre les nations». En tant que sépharades dont les aïeux ont été accueillis par l'Empire ottoman, Elias et sa mère voyagent avec des passeports turcs. Or, endosser l'identité turque, en ces temps troubles, c'est devenir un ennemi potentiel pour les douaniers roumains ${ }^{6}$, d'où la scène qui suit:

[Comme] les vivres se faisaient déjà rares à Vienne après le premier hiver de guerre, [ma mère] fit provision de légumes secs avant de partir. On enfila d'innombrables graines de diverses légumineuses; on en emporta une valise pleine et ma mère se trouva fort marrie quand les douaniers roumains de Predeal, à la frontière hongroise, déversèrent tout le contenu sur le quai. Le train se mit en marche, ma mère bondit de la banquette, mais juste pour voir les douaniers ricanant et nos trésors, y compris la valise, répandus sur le quai. [...]

D'après elle, c'était à cause de nos passeports turcs que les douaniers roumains s'étaient comportés de cette façon. [...] Ma mère affirma que les Roumains se seraient à coup sûr conduits différemment si elle avait voyagé avec son passeport de jeune fille, sous le nom d'Arditti. Ils avaient un faible pour les Italiens parce que leur propre langue venait de là-bas. Mais c'étaient les Français qu'ils préféraient. (Canetti, 1985: 123 sq)

Canetti s'amuse alors: quelle belle guerre que celle-là, sur le dos de Turcs qui n'en sont pas, et par l'entremise de graines et de valises! Après

5 Le cadre d'énonciation varie: parole en différé pour Canetti en 1977, et parole quasi simultanée aux événements pour Yovkov. La lecture comparée devient alors un moteur d'interprétation original, qui empêche la récupération de Yovkov par le discours national: en mettant côte à côte un écrivain bien plus "détaché» de la Bulgarie que son confrère ne l'est, la lecture croisée avec Canetti redonne aux écrits de Yovkov une perspective plus universelle.

6 En 1915, l'Empire Ottoman et l'Autriche-Hongrie sont du côté des Empires Centraux. La Roumanie, d'abord neutre, ralliera l'Entente, camp opposé. 
avoir fait le tour des nationalités en guerre, (fût-ce en temps de paix: les "préférences»), Canetti conclut:

Je venais d'un pays en guerre [Autriche-Hongrie] mais [...] c'est pendant ce voyage de retour que je commençai à comprendre que la haine entre les nations est un phénomène universellement répandu. (Canetti, 1985:123sq nous soulignons)

Décidément, il n'est pas aisé le métier du passeur de frontières!

Cependant, grâce à cette lecture croisée, le drame d'un pays séparé d'un bout de son territoire dépasse les limites étroites d'une querelle de frontières entre voisins en guerre, pour devenir ce qu'il est, en son essence, une tragédie humaine:

Il m’a volé l'oreille gauche. Je lui ai arraché l'œil droit. Il m’a escamoté quatorze dents. Je lui ai cousu les lèvres. Il m'a rôti le derrière à point. Je lui ai retroussé le cœur. Il a mangé mon foie. J'ai bu son sang. - Guerre. (Canetti, 1985: 975, italiques de l'auteur).

Canetti est un graveur à vif: sans avoir fait la guerre, il croque son rictus à traits hachurés, dans un cuivre rongé par l'acide. Accumulation enragée de verbes de l'arrachement: telle est la grimace hideuse de la guerre. Tous ces morts, tombés... sans nulle autre raison que la déraison humaine!

S’il fallait trouver la correspondance de cette gravure acérée dans la peinture à l'huile, on hésiterait entre Goya, Bosch ou Georg Grosz. Ou peutêtre évoquerait-on la figure déchaînée, colorée, monstrueuse, de cet Ange du foyer que Max Ernst peignait en 1937, en pleine Guerre d'Espagne.

\section{L'art du décentrement du regard: subversion, superposition}

Dès sa chronique liminaire, «Les Sans-patrie», Yovkov s'inscrit et se distancie tout à la fois par rapport à son récit. De ces malheureux «sanspatrie », le chroniqueur partage le sort en tant qu'homme, et se sent dans un premier temps solidaire. Comme eux, il se retrouve à errer dans la capitale, son regard glisse dans les leurs, regards lourds et perdus au loin, ayant pour seule flamme l'injustice et la douleur, pesants comme seule sait peser l'absence:

Il y a, boulevard Dondoukov [à Sofia], un petit café: le Dobroudja. [...] Ici, ce sont toujours les mêmes personnes qui viennent, et elles viennent avec la même régularité et constance qu'auraient eues les membres d'une société de conspiration se rendant à leur club, ou les malades d'une même maladie qui se réunissent dans le cabinet de leur médecin. [...] dans ce café, des Dobroudjans [faisaient] leurs réunions. Ils avaient besoin de prendre 
certaines décisions. Je ne suis pas allé à ces réunions. Mais j'avais, malgré tout, un désir sans pareil de me rendre là-bas, peu importe le moment, quitte à ce que ce soit en dehors des réunions. (Yovkov, 1956, t. 2: 53, nous traduisons et soulignons).

Certes, les «sans-patrie» sont décrits comme des «mauvaises herbes ${ }^{7}$ » (Yovkov, 1956, t.2; 55-56 ), des racines arrachées qui n’avaient pas à être là, et que seul traîne désormais le vent.

Pourtant, la solidarité de l'homme Yovkov (banni aussi) s'arrête là: chez l'écrivain, ces réunions entre exilés font naître un malaise. Ce qu'il aimerait, lui, c'est tout simplement se retrouver en ce lieu qui porte le nom de la patrie perdue: du seul fait qu'il s'appelât ainsi, le café Dobroudja fait revivre, en plein cœur de Sofia, la province danubienne perdue ${ }^{8}$. Seul le nom enchanteur du café Dobroudja attire Yovkov. Car il le sait: l'immense tristesse qu'il éprouve, parmi ces «bannis par soustraction (de territoire)», ne peut être... qu'un deuil privé 9 .

Reste à savoir comment, contemporains à ces époques, Yovkov et Canetti en viennent-ils à secrètement subvertir la logique militaire et la machine idéologique qui les sous-tend.

Malgré le parti pris d'ancrage dans l'Histoire immédiate bulgare, les Chroniques 1912-1918 de Yovkov jouent sur la superposition d'une infinité de temps, d'époques, de civilisations, échappant aux limites temporelles de leur titre, pour donner une perspective inattendue - grandiose ou résignée à ce qui se passe sous nos yeux: un exploit légendaire.

Yovkov dépasse ainsi cette ambition modeste: couvrir les guerres balkaniques côté bulgare uniquement. Tantôt fait-il mine d'explorer les temps récents, mais le voici qui élargit la référence à l'histoire bulgare plus générale, voire à l'histoire de l'Europe sur ces mêmes lieux et à l'Empire romain. Tantôt s'attarde-t-il sur le paysage de la Dobroudja, mais le voici qui plonge, soudain, dans les profondeurs archéologiques du lieu, faisant surgir ses couches immémoriales.

7 Nous retrouvons la même image des mauvaises herbes arrachées dans Les Chardons $d u$ Baragan de Panaït Istrati.

8 Le passage textuel Dobroudja/ Sofia s'effectue grâce au mot mitoyen «auberge", krătchma, correspondant provincial des cafés sofiotes.

9 Canetti n'approuvait guère, lui non plus, ce type de réunions: il esquivait toute clôture que pouvaient dessiner ses coreligionnaires juifs, y compris leurs propres divisions internes (Juifs d'Orient/ Juifs d'Occident). 
Ainsi, le lieu se compose simultanément de toutes ses époques, y compris futures: la Dobroudja des Chroniques, est cet ensemble de couches spatiotemporelles superposées.

La conséquence de cette traversée des frontières du temps et de l'espace est importante: elle permet au récit yovkovien d'enjamber en même temps les frontières nationales. Et même si, par ailleurs, les références roumaines/ bulgares demeurent dans le texte, ce qui le porte réellement à se dépasser luimême, c'est bien son élan humaniste, coulé dans une description qui survole les conflits nationaux du moment.

Comme si une tout autre vision se superposait à la réalité des combats, l'évinçait même, et nous voilà plongés dans une atmosphère festive où langues et cultures étrangères se retrouvent: dans «Dobroudja autrefois", en décrivant la «bigarrure inimaginable» des foires, Yovkov se réjouit du Babel de langues qu'il y avait autrefois entendu, support d'une diversité humaine foisonnante.

La guerre semble s'être évanouie, et à sa place, c'est la cohabitation heureuse de nationalités diverses en un même lieu qui ordonne la narration yovkovienne. L'évocation des couleurs éclatantes des tenues vestimentaires participe ainsi d'un émerveillement infini devant les temps de paix, une époque qui, contrairement à la présente, ne dressait pas les étrangers les uns contre les autres, mais les regardait vivre ensemble, traversant les frontières sans entraves.

Cet amour de l'étranger (que d'aucuns jugeraient «déplacé» en temps de guerre) affleure également dans «Koubadine», chronique que Yovkov écrit en pleine offensive bulgare dans la Dobroudja annexée (1916). En pénétrant dans le village de Koubadine (Medjidié), c'est bien vers un "homme venu d'ailleurs » que Yovkov se dirige - un vieux sage né en Crimée et installé sur ces terres.

On dirait que les combats n'existent pas, car Yovkov interroge le vieux Tatare du village... sur l'origine du nom de Koubadine! Et alors, oublieux des hostilités alentour, Yovkov et son interlocuteur s'adonnent au plaisir tout oriental du couple conteur/ auditeur. Tandis que le vieil homme raconte, le chroniqueur nous restitue sa parole: "[Le nom de Koubadine] provient de deux mots arabes: houbd, qui voudrait dire «nœud» ou "pôle», et edi «sept». Koubadine veut dire noud de sept chemins». Ce village n'a donc jamais été une frontière infranchissable, mais un lieu de passage pour les peuples méditerranéens fort prisé: «Il y avait eu ici (et il y est resté) un grand croisement de chemins: des routes comme Dobritch-Medjidié, Dobritch- 
Constanza, Silistra-Constanza et tant d'autres encore s'entrecroisaient précisément ici.» (Yovkov, 1956, t. 2: 58, nous traduisons).

Et Yovkov de rappeler que le village au double nom Koubadine / Medjidié doit son nom au sultan ottoman Abdul Medjid (1823-1861) - encore un étranger présumé ennemi que l'écrivain réhabilite pour l'occasion. En écoutant le vieux Habbibullah évoquer la «splendide magnificence du passé», l'assentiment de Yovkov se fait sentir. Non seulement il ne s'érige pas en juge, mais il reconnaît la «place dans l'Histoire bulgare» qu'ont eue ces sultans ${ }^{10}$.

Venant de Yovkov (descendant bulgare des populations dominées par l'Ottoman), ce récit rapporté œuvre pour la dissolution du ressentiment comme fondement psychique de la mémoire, et l'incline vers un désir de dialogue avec l'étranger. Avec tout étranger ${ }^{11}$. La plume du chroniqueur du temps présent déjoue ainsi la logique militaire des frontières interdites, et lui substitue des images de frontières qui invitent au voyage.

Mieux même: en traitant librement la ligne de passage au cœur même des offensives militaires (et de l'exhortation au chauvinisme qui leur est propre), Yovkov parvient à enjamber jusqu'au cadre même dans lequel ses Chroniques s'inscrivent.

Enfin, c'est par un élargissement de la notion de frontière que procède Yovkov dans À l'ancienne frontière.

Il est vrai que cette chronique s'attarde sur une figure pathétique: un vieil homme bulgare, demeuré sur les territoires annexés, qui regarde au loin les terres de son ancien pays et en rêve. Du seul fait qu'une nouvelle frontière a été tracée, l'homme éprouve le territoire même où il se trouve comme étranger, seul «l'autre côté» étant à présent désirable:

Même l'air là-bas est différent, et le soleil brille plus fort, et le ciel est plus beau et plus bleu. [...] Et les hommes aussi sont bons, là-bas, toujours souriants, heureux. [...] Autrefois ici aussi c'était pareil, mais comme si cela n’avait jamais été, comme si c'était un songe. (Yovkov, 1956, t. 2:58, nous traduisons)

Le vieil homme fait d'abord l'expérience inquiétante d'une terre évanescente, ce «ici aussi» que l'annexion fait basculer dans le «songe», lors

10 Grâce aux souvenirs du Tatare, Yovkov rappelle que le village doit son essor à ce sultan: ayant trouvé la bourgade «insalubre et boueuse», Abdul Medjid avait «ordonné que l'on creuse des canaux vers le Danube» afin d'éconduire les eaux limoneuses.

11 «Les hommes de la Dobroudja ne sont pas de Dobroudja» rappelle fort à propos Inna Peleva, ils viennent tous d'ailleurs.» (Voir Peleva, 2000). 
même qu'il s'agit du même lieu que la veille. À peine le lopin de terre est-il devenu roumain, que voici son soleil terni, son ciel assombri, son air vicié.

Néanmoins, précisément par ce glissement des références spatiales, la note patriotico-nostalgique sera imperceptiblement dépassée dans la narration. Yovkov n'ouvrait-il pas cette même chronique par une apologie de la vie près des frontières en général - la ligne d'horizon de la mer, ou la ligne-frontière:

Ceux qui n'ont jamais connu le bonheur [...] de voir, une fois au moins de leur vie, la mer ou une frontière, gardent toujours au plus profond de leur âme des pressentiments obscurs de quelque chose d'énigmatique, d'étrange. [...] Parce qu'un homme qui se tient au bord d'une falaise escarpée [...] ou sur cette ligne-frontière coupée net [...], là comme ici, il se tient également devant une énigme, un secret (Yovkov, 1956, t. 2: 58, nous traduisons).

C'est un «bonheur» que d'avoir vécu une vie près des berges, des frontières, qui sont autant de "fenêtres grand-ouvertes" sur une connaissance simultanée de l'ici et de l'ailleurs, de ce côté-ci et de l'autre! Grâce à la comparaison frontière/mer, les espaces pourtant éprouvés par la guerre, ne sont plus limites, mais horizon - nouvelle démonstration du tour de force yovkovien, de son art du décentrement du regard.

Mais chaque lieu évoqué sera désormais marqué par cette soif dévorante, soif d'une altérité de la pensée, qui récuse l'hostilité et s'emploie à la dépasser. Pour Yovkov, comme pour Canetti, ce n'est plus le lieu qui importe, mais le mouvement même de la traversée - nécessaire, inévitable - d'une frontière: le sort de la plaine du Danube n'est qu'un de ses paradigmes littéraires.

\section{Dynamique du mélange des espaces}

En 1928 et 1929, Canetti (installé à Vienne) fait deux séjours à Berlin. Le territoire intime qui se dessine alors est étrange. C'est un lieu symbolique composé de plusieurs: Odessa, Varna, Danube, mer Noire... Car le Berlin de Canetti est vu à la lumière des Récits d'Odessa de l'écrivain russe Babel, tant et si bien qu'il n'y a plus de frontière entre les lieux affectionnés, et que le franchissement de tous les espaces devient synonyme - comme chez Yovkov - d'ouverture.

Dans ce Berlin réinventé à la lumière des Récits d'Odessa de Babel, un espace inédit se dessine. Il n'est que symbolique, parce que les paysages intimes, même les plus éloignés géographiquement, peuvent s'y rencontrer: 
Ce fameux voyage qui domina les rêves de mon enfance, [la descente et la remontée du fleuve de Vienne à Odessa et d'Odessa à Vienne et Roustchouk] aurait trouvé sa juste place dans cet itinéraire.

J'étais curieux de connaître Babel, comme s'il était venu de cette région dont la moitié de mon cour se réclamait. Je ne me sentais rassuré que dans un lieu ouvert sur le monde. Odessa était l'un de ces lieux. Babel l'avait ressenti ainsi dans ses Histoires. Dans la maison de mon enfance, toutes les fenêtres s'ouvraient sur Vienne, et maintenant, dans une façade jusque-là ignorée, une seule sur Odessa (Canetti, 1985: 599sq).

Odessa est ainsi une fenêtre qui s'ouvre sur la «façade ignorée» d'un Berlin au départ oppressant, partagé entre émerveillement et hostilité. De même que l'enfance à Roustchouk avait toujours gardé une porte entrebâillée sur Vienne - mot magique, le premier mot allemand que l'enfant Elias sût -, la visite à Berlin faite par un jeune homme peu sûr encore de son avenir d'écrivain, trouve son ouverture toute naturelle sur un paysage russo-bulgare.

Néanmoins, si la «maison de l'enfance» est ici évoquée, elle ne marque pas la fin d'un itinéraire, mais la nécessité d'un itinéraire autre, ouvert sur un espace symbolique indispensable:

J'avais entendu parler d'Odessa dès ma petite enfance, ce nom remontait à la toute première partie de ma vie. Je prétendais avoir des droits sur la mer Noire, sans l'avoir vue plus de quelques semaines à Varna. La couleur, la force, la brutalité des histoires de Babel sur Odessa étaient comme nourries de mes propres souvenirs d'enfance; sans le savoir, j'avais trouvé chez lui la capitale naturelle du cours inférieur du Danube et il m'aurait semblé normal que la ville d'Odessa se trouvât à l'embouchure du Danube (Canetti, 1985: 599 sq).

Et c'est alors qu'une fenêtre s'ouvre, sur un paysage en surimpression, où le Danube et la mer Noire peuvent même s'y rencontrer, tant la mémoire affective se moque de la cartographie réelle, sur laquelle pourtant elle s'appuie.

La co-présence de toutes ces villes au cour de ce seul espace de Berlin est une création de Canetti qui nous subjugue à force de dépaysement. La portée familière que Canetti finit par reconnaître à Berlin, non seulement récuse toute définition du familier comme un lieu de résidence, mais affirme l'invention d'un chez soi précisément dans et par le détour que la passion de l'altérité engage.

Le «métier du poète», si cher à Canetti, ne commence-t-il pas précisément avec l'abandon de l'assurance que procure l'espace familier, et avec 
l'élection d'un familier de l'écriture, qui ne surgit qu'au cœur d'un désiré dépaysement?

Ce désir d'éloignement qui finit par rendre familiers les espaces inquiétants chez Canetti, a pour pendant, chez Yovkov, une pratique inversée : le dépaysement du familier.

\section{Le dépaysement du familier chez Yovkov}

Lorsqu'en 1920 Yovkov choisit l'émigration économique à Bucarest, il renoue avec sa veine d'avant-guerre, celle de "La Plainte du berger». Résidant sept ans durant de l'autre côté de la frontière, il rédige ses célèbres Légendes de la Stara Planina (1927), qui ont pour lieu d'action Jéravna. Mais c'est une Jéravna métamorphosée, réinventée. Car, si les guerres ont pris fin, c'est un interdit intime qui pousse Yovkov à ériger une barrière physique entre lui et son village natal: appréhendant un retour réel sur les lieux de son enfance, ses descriptions de la montagne seront une écriture de mémoire et d'invention mêlées.

L'espace affectif yovkovien, nous l'avons vu, se partage entre Jéravna (la montagne) et Tchifoutköy (la plaine de Dobroudja), deux patries également marquées par l'absence: la Dobroudja, passée roumaine, lui est interdite, et l'écrivain ne revisitera plus Jéravna. Hissées au rang du mythe, dont la principale caractéristique est la répétition-variation, la plaine et la montagne se croisent et se confondent, dans les Légendes, quand elles ne se relayent pas.

Dès son premier récit: La Plainte du berger, Yovkov recrée la distance séparant le haut et le bas, le lointain et le proche pour la seule localité de Jéravna. Lorsque le joueur de flûte Stéphane fait le choix de son bannissement du village comme réponse à l'injustice de l'espace social, il instaure la chaîne du Balkan comme un mur (in)franchissable ${ }^{12}$ : le Balkan, c'est son lieu où personne ne le retrouvera, d'où il ne redescendra pas. Seule sa musique survole désormais l'espace.

Les lieux des Légendes se caractérisent par une mobilité dans l'espace du texte - un jeu sur la désignation qui leur procure un non-lieu géographique. Quand la réelle Jéravna, jamais nommée ainsi, mais par son nom d'autrefois (Jérouna), devient sans se démentir tous les lieux-synecdoques qui la

12 Dans l'imaginaire yovkovien, c'est toujours le mur du Balkan qui joue le rôle du séparateur, celui du cadre à franchir. 
dessinent en creux, Yovkov signe des pages où la variation procède de la réinvention des lieux.

Tenue physiquement à distance, Jéravna demeure (comme il le confie) une «mythologie fantastique, effrayante». Yovkov nomme les lieux pour mieux jouer de leur flottement et de leur décentrement. Par ses couches superposées de noms, l'espace réel perd ses repères: il est volontiers brouillé et en même temps mobile. Le même et l'autre du lieu deviennent ainsi moteurs de la création, de l'élaboration mythique, la seule qui, en embrassant les pôles opposés de l'espace symbolique, peut dire l'étrangeté qui survient sur le lieu-même du familier.

Tous les noms de lieux ont une vie propre dans l'univers des Légendes, une vie animée et mise en perspective dans chacun des textes. Koukov vir («Bojoura») a les frontières verticales brouillées, le lecteur a tantôt l'impression que le trou d'eau se trouve en haut (la forêt, les rochers), tantôt en bas (les champs, la rivière). Jouant sur le haut et le bas tour à tour, les lieux revêtent le caractère mythique de ce qui est à la fois partout et nulle part, et qui peut ainsi s'élever au statut d'un espace-temps particulier, pouvant tout aussi bien s'incarner dans ces lieux que dans d'autres... Ainsi, écrivant depuis Bucarest, Yovkov charge le lieu natal d'une dynamique de mélange des espaces (voir aussi Raseva/ Semerdzieva: 1996).

Étrangement, Ouroum Eniköy (autre nom déguisé pour Jéravna) entre en résonance avec Tchifoutköy ${ }^{13}$, le village dobroudjan. Un village nommé Ouroum Eniköy existait bien, mais il se trouvait... sur la côte sud du littoral bulgare! Seule donc la proximité sonore Ouroum Eniköy/ Tchifoutköy justifie ce choix de Yovkov: n'aurait-il pas joué à situer dans le Balkan... un lieu qui lui rappelât en même temps les deux lieux perdus de vue, celui près du Danube, et celui niché au cœur du Balkan?

Mais en cela, sommes-nous bien loin de Roustchouk, affectivement présent dans le Berlin de Canetti via Odessa?

Le «métier du poète» se dessine ainsi toujours en creux dans de semblables paysages de l'intimité réinventée, espaces poétiques où l'improbable devient le seul réel, et où la carte géographique est recréée par une incandescence plus que réelle de l'intimité assoiffée. Reprises et variations constituent le matériau disponible pour une navigation désentravée.

13 Aujourd'hui, il porte le nom de l'écrivain, Yovkovo. Mais quand Yovkov écrit ses Légendes, Tchifoutköy appartient à la Dobroudja annexée. 


\section{Un « territoire de l'homme» : conclusion et ouvertures}

La fenêtre, l'ouverture, la possibilité incessante d'un ailleurs qui, se dessinant comme le ruban d'un fleuve, relaye un ici autrement appauvri: autant de conditions, pour Canetti, dans la manière dépaysée qu'il a d'habiter les différents espaces (aimés ou craints), les ramenant dans le seul instant de l'écriture. Berlin, ce n'est plus seulement Berlin, mais toute une fresque multicolore, un territoire où éclot la poésie comme création: en ce Berlin-là, il n'est même pas exclu que coule le Danube, ou encore les eaux mouvementées de la mer Noire près d'Odessa.

Constamment animée par le souci de l'humanité perdue, la parole du poète n'évoque les frontières que pour en dénouer les barbelés. Et si la pensée de Canetti résiste à l'air du temps, c'est parce qu'elle garde en elle la conscience des enjeux de l'Histoire (et cette conscience est la promesse d'une rencontre possible).

Sans doute parce qu'il est juif, l'humaniste Canetti prévoit d'autant plus crûment la dérive dans laquelle la mémoire blessée peut entraîner celui qui choisit de poser sur elle les bases de sa nouvelle maison. C'est parce qu'on a porté atteinte à l'humanité dans l'hommeque l'écrivain s'adresse à ses contemporains, non pour entretenir la mémoire de la barbarie (subie, infligée), mais pour bâtir ce que Régine Robin appelle une «mémoire critique», inlassablement tendue vers une humanité à reconquérir (Robin: 2003).

Dès l'instant où il envisage son Histoire contemporaine non pas comme la réitération de conflits ancestraux, mais comme la recherche inépuisable d'alternatives de dialogue, la sensibilité d'un auteur s'inscrit dans le sillage du Dichter, le poète gardien des métamorphoses, le créateur d'espaces.

Cette vue plus large qu'ont Yovkov et Canetti est-elle due à la coloration multiethnique de leurs premières années au bord du Danube? Toujours est-il que l'espace auquel ils aspirent tous deux est celui d'un territoire de l'homme.

\section{Bibliographie des ouvrages cités}

Canetti, Elias, 1985, Écrits autobiographiques, Paris, «La Pochothèque». Comprend les trois tomes de l'autobiographie de Canetti: La Langue sauvée, Le Flambeau dans l'oreille, Jeux de regard, de même que les Réflexions des années 1942-1985: Le Territoire de l'homme (1942-1972) et Le Cour secret de l'horloge (1973-1985). 
Handke, Peter, 1987, Images du recommencement, Paris, Bourgois.

Yovkov, Yordan, 1956, Letopis 1912-1918, Sofia, Bălgarski pisatel, t. 2.

—, 1956, Staroplaninski legendi, Sofia, Bălgarski pisatel, t. 3.

—, 1999 (trad. Marie Vrinat) Légendes du Balkan, Paris, L’Esprit des Péninsules.

Kuzmanova, Antonina, 1989, Ot N»oj do Krajova: V»pros»t za Uzna Dobrudza v mezdunarodnite otnoseniâ (1919-1940), Sofia, éd. Nauka i izkustvo [De Neuilly à Craïova: la question de la Dobroudja du Sud dans les relations internationales (1919-1940), Sofia, éd. Science et Art].

Peleva, Inna, 2000, «Jordan Jovkov. Domăt na čuždeneca» («Yordan Yovkov. Le Foyer de l'étranger»), in Bălgarskata literatura - figuri na četeneto, Sofia, Figuri. Raseva, Canka/ Semerdzieva, Galina, 1996, "L'interaction des toponymes avec les autres couches lexicales", in Pogled v jovkovija svijat, Dobrič.

Robin, Régine, 2003, La mémoire saturée, Paris, Stock. 